\title{
Subungual Nail Erythrasma Presenting as Melanonychia: A Rare Finding
}

\author{
Jalal Maghfour ${ }^{a}$ Jennifer Kane ${ }^{b}$ Leslie Robinson-Bostom ${ }^{b}$ John Kawaokab \\ Nathaniel Jellinek ${ }^{b}$ \\ aTulane Medical School, Tulane University, New Orleans, LA, USA; ${ }^{b}$ Dermatology, Warren Alpert Medical School of \\ Brown University, Providence, RI, USA
}

\section{Established Facts}

- Erythrasma is a well-known, common superficial bacterial infection of intertriginous sites.

- Erythrasma with involvement of the nail plate is a rarely reported finding in the literature.

- Nail erythrasma may present clinically as nail plate dystrophy and subungual hyperkeratosis.

\section{Novel Insights}

- Nail erythrasma may also present clinically as melanonychia.

- Nail erythrasma may self-resolve gradually without intervention.

\section{Keywords}

Erythrasma $\cdot$ Cornyebacteria $\cdot$ Nail matrix $\cdot$ Nail plate

\section{Abstract}

Nail pathology may reflect a wide array of localized and systemic dermatological conditions. Certain nail findings such as melanonychia can create diagnostic challenges even to nail experts. We report a case of a 78-year-old man who presented with melanonychia of the great toe. Nail clipping showed focal melanin deposition, and dermoscopy demonstrated a region of localized erythema in the lunula concerning for possible melanocytic neoplasm. Subsequent nail biopsy showed numerous vertically oriented filamentous bacteria and coccobacilli within the nail plate consistent with a

karger@karger.com

(C) 2020 S. Karger AG, Basel

www.karger.com/sad

Karger ${ }^{\prime}=$ diagnosis of subungual nail erythrasma. Nail erythrasma is a rare entity. Additionally, this case highlights a new clinical presentation of nail erythrasma as melanonychia.

(c) 2020 S. Karger AG, Basel

\section{Introduction}

Erythrasma is a common, superficial, cutaneous bacterial infection of intertriginous and web spaces commonly caused by Corynebacterium minutissimum, a Gram-positive rod [1]. Erythrasma with involvement and invasion

Jalal Maghfour and Jennifer Kane contributed equally to the study. 

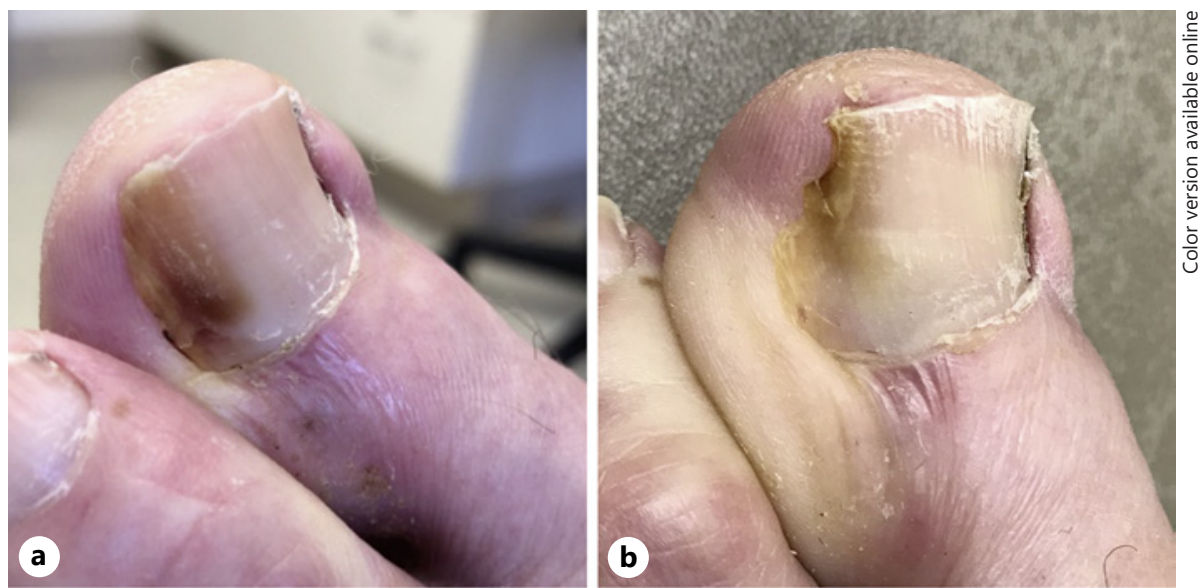

Fig. 1. a Smudged brown proximal lateral nail plate discoloration. b Residual minimal brown lateral nail plate discoloration, limited to the distal plate, at follow-up visit. c Wood's lamp examination showing coral red fluorescence between the left first and second toe web space. d Wood's lamp examination showing mild fluorescence of the proximal lateral nail plate.
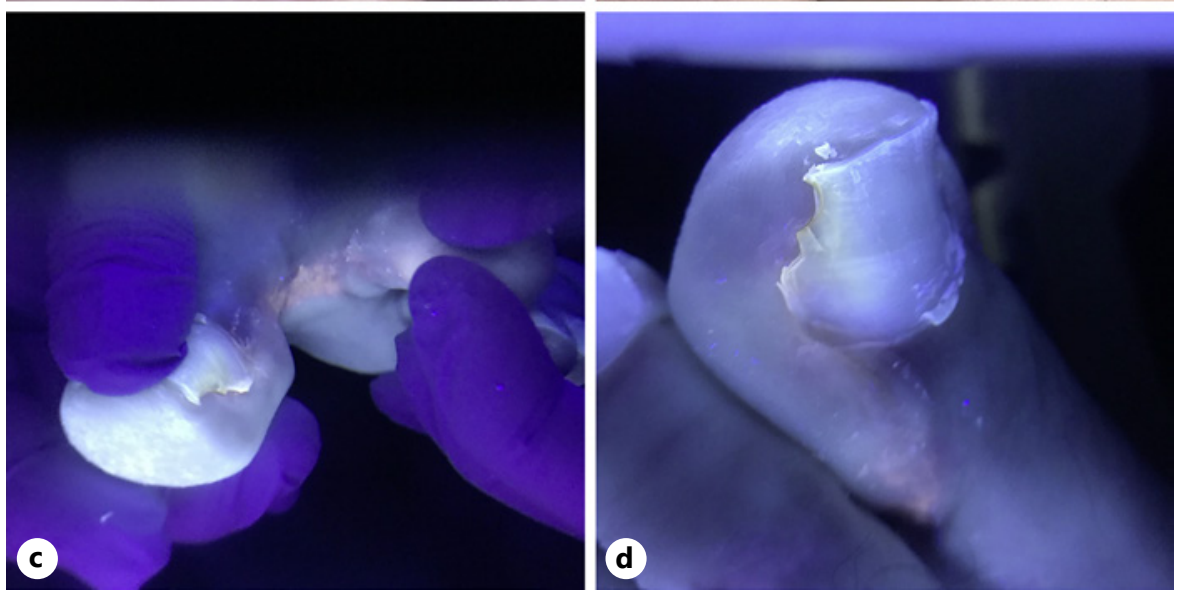

into the nail plate is a rare entity that is not well described in the literature. In the few reported cases, it presented with nail plate dystrophy and hyperkeratosis clinically, resembling onychomycosis [2]. We describe a case of subungual nail erythrasma with a unique clinical presentation of melanonychia.

\section{Case Report}

A 78-year-old man with a history of cutaneous squamous cell carcinoma and psoriasis presented to the dermatology clinic for his routine full skin exam. Melanonychia involving the left great toe lateral nail plate was identified. The patient was unable to determine the exact onset but noted it had been present for several months without noticeable change. The lesion was asymptomatic, and there was no known history of trauma. He denied associated joint pain or swelling, family history of similar lesions, or melanoma. A nail clipping was performed, which showed nail plate and subungual keratin fragments with surface bacteria. The Fontana-Masson stain was positive for focal melanin deposition within the nail plate onychocytes, and PAS-D was negative for yeast or hyphal elements. The patient was subsequently referred for further evaluation. Nail examination revealed smudged brown proximal lateral nail plate discoloration (Fig. 1a), with dermoscopy highlighting a red area of origin in the matrix; there was no periungual pigmentation. There was no malodor or associated pits seen on examination of the plantar feet. Initial clinical impression was subungual hemorrhage; however, the presence of focal melanin seen on initial clipping and proximal red lunula discoloration raised concern for a possible melanocytic lesion including melanoma. Punch biopsies of both the nail matrix and nail plate were performed. Histopathology revealed hyperkeratosis and parakeratosis of the nail bed with the attached nail plate (Fig. 2a). Numerous vertically oriented filamentous bacteria and coccobacilli were observed within the superficial nail plate (Fig. 2b). These morphologic patterns were highlighted using Gram stain (Fig. 2c, d). The SOX10 stain was negative in the matrix epithelium. These findings were diagnostic of subungual erythrasma. The patient's nail subsequently improved without further intervention. At his follow-up visit 6 months later, there was only minimal smudged brown pigmentation remaining (Fig. 1b). Wood's lamp examination revealed coral red fluorescence between the left first and second toe web space (Fig. 1c); the nail unit showed mild fluorescence (Fig. 1d). 
Fig. 2. Nail bed with attached nail plate showing mild hyperkeratosis and parakeratosis with focus of superficial detachment. a $H \& E$, original magnification $\times 20$. Highpower photomicrograph showing numerous vertically oriented filamentous bacteria and coccobacilli within the superficial nail plate. $\mathbf{b} H \& E$, original magnification $\times 100$. c H\&E, original magnification $\times 400$. Gram stain highlighting numerous vertically oriented filamentous bacteria and coccobacilli within the superficial nail plate. d H\&E, original magnification $\times 400$.
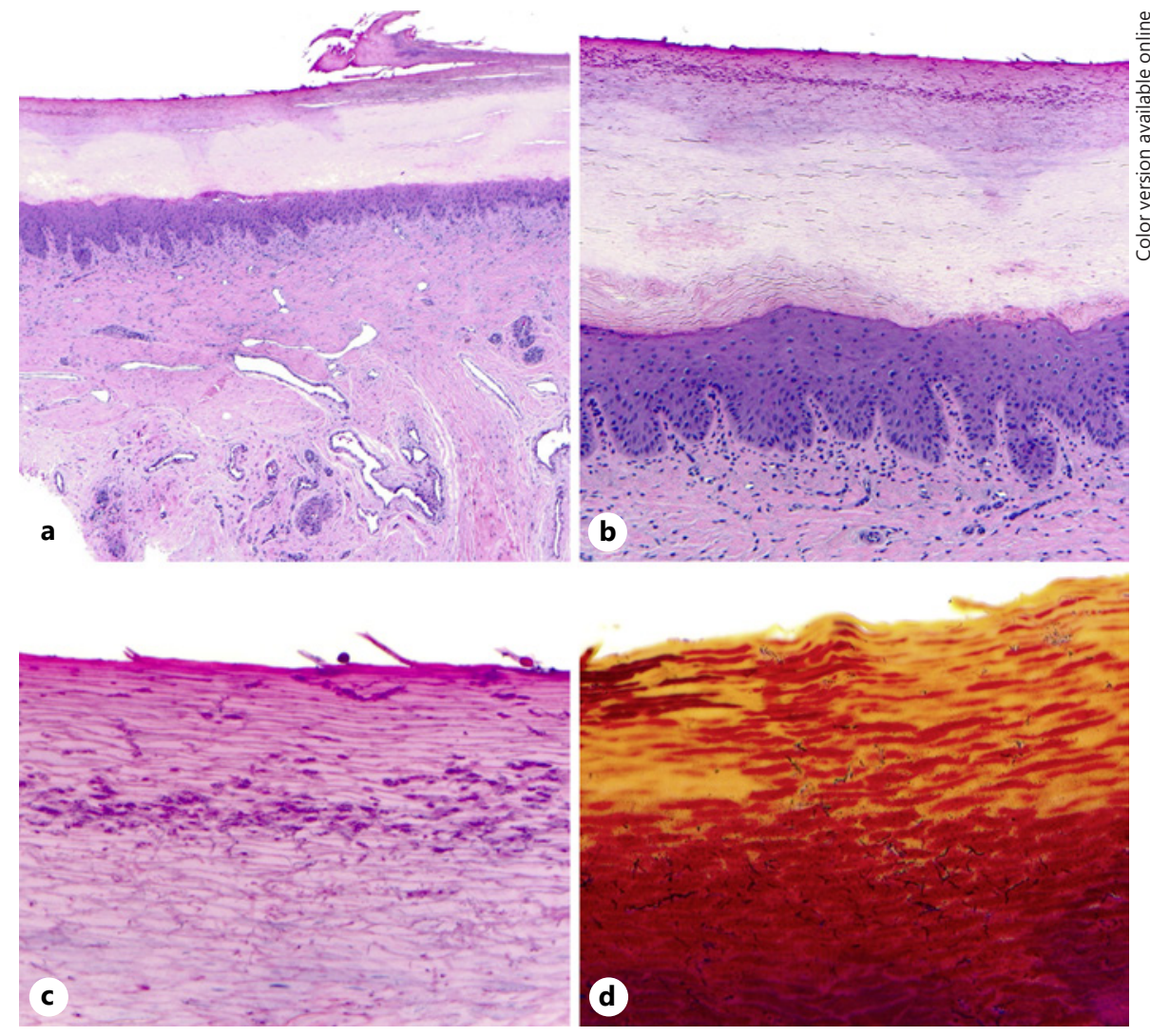

\section{Discussion}

Nail erythrasma as a clinical entity has been rarely reported in the literature. In the few reported cases, it has been described to present with similar clinical features of onychomycosis, such as yellow discoloration with nail plate dystrophy and subungual hyperkeratosis [1]. To the best of our knowledge, this is the first reported case of nail erythrasma presenting clinically as melanonychia and presenting with signs that overlap with nail melanoma.

Melanonychia can result from either a process of melanocytic activation or melanocytic proliferation. While melanocytic activation can be a physiologic process in darker skin individuals, it has many alternative etiologies, including trauma and infection [3]. Melanocytic activation following infection can result in melanin synthesis with a subsequent deposition of melanin-rich melanosomes in the differentiating nail matrix cells [4]. It is possible that corynebacterial infection of the nail plate induces inflammation, resulting in activation of melanocytes and subsequent melanonychia as seen in this case.

Nail erythrasma can be caused by various pathogens, such as C. minutissimum, Nocardia species, and Kytococ- cus sedentarius. In a report of 17 patients, the causative agent was found to share common physiologic and morphologic patterns with corynebacteria and Nocardia. H\&E-stained sections often show coccobacilli and occasional filamentous forms in the superficial stratum corneum. The organisms are highlighted with Gram, periodic acid Schiff (PAS), GMS, and Giemsa stains [1]. As illustrated in Figure 2a-d, the histopathologic features of this case are consistent with the patterns reported in the literature.

Cornyebacteria are Gram-positive rods and members of normal cutaneous flora, which can become pathogenic when precipitated by warm, moist local environments. Both Corynebacterium species and K. sedentarius are known to cause infections on acral surfaces. Pitted keratolysis presents clinically as crater-like pits on the plantar feet and toes, often associated with excessive sweating and malodor [5]. While risk factors for nail erythrasma are not completely understood, it has been suggested that pitted keratolysis may predispose to the development of nail erythrasma [6]. In our case, the patient did not have evidence of pitted keratolysis; however, he was found to have concomitant erythrasma of the toe web spaces, which we 


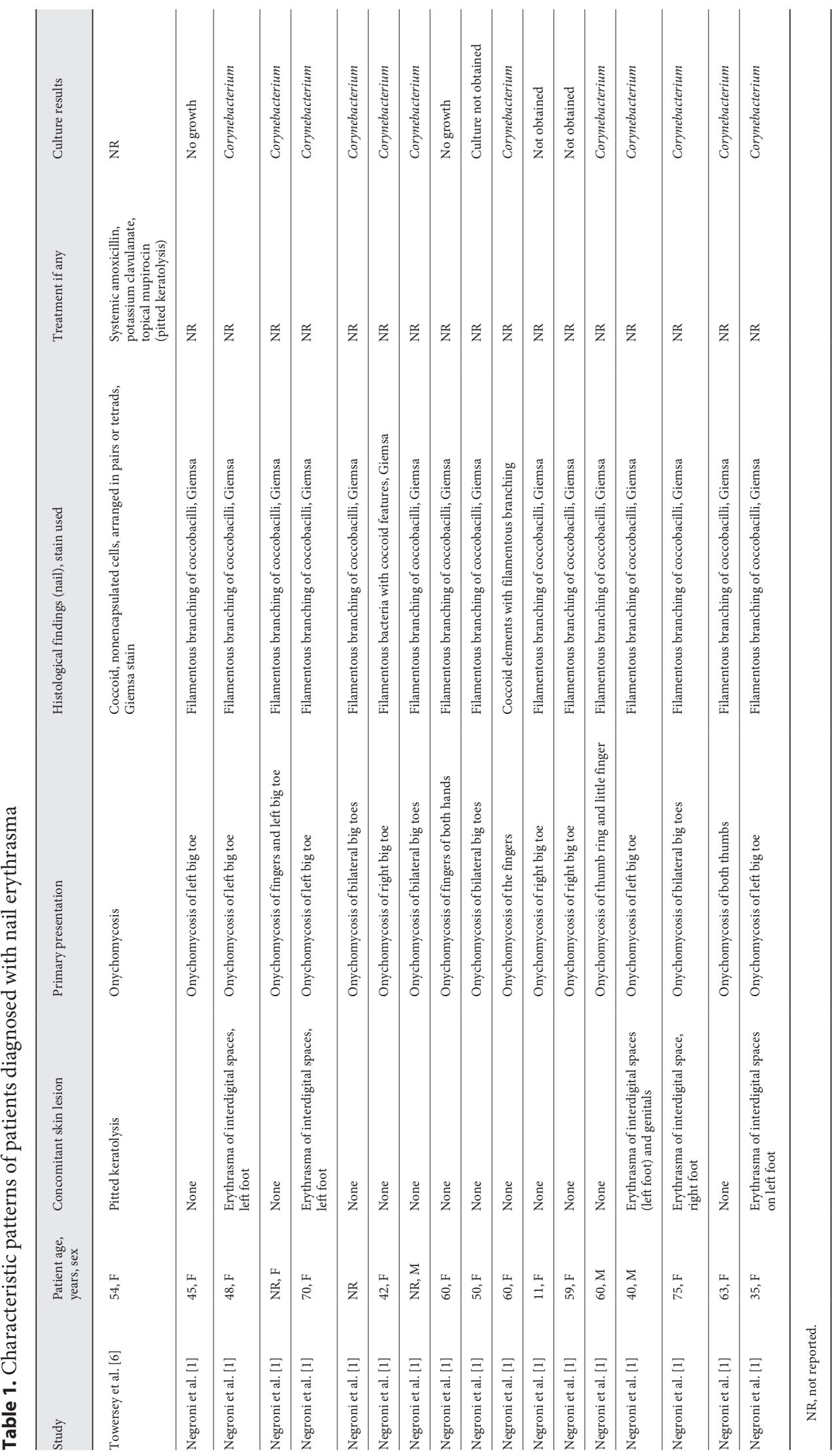


hypothesize is a likely additional predisposing factor to the development of nail plate involvement.

While there are no clear treatment guidelines for nail erythrasma in the literature, Towersey et al. [6] successfully reported clinical resolution of nail infection due to $K$. sedentarius with a course of oral amoxicillin and potassium clavulanate (Table 1). Sulfate gentamicin solution was also used for treatment of localized nail lesions. In our case, the patient experienced spontaneous clinical improvement following biopsy.

Nail erythrasma is a rare finding. Our case highlights that corynebacterial infiltration into the nail plate can present clinically as melanonychia. It is therefore important to increase awareness of this entity and broaden its clinical presentation to include melanonychia.

\section{Statement of Ethics}

The patient has given his informed consent to publish the photos and details of the case.

\section{Conflict of Interest Statement}

The authors declare no conflict of interest.

\section{Funding Sources}

The authors did not receive any funding.

\section{Author Contributions}

Jalal Maghfour, MS: helped in design, write-up, and manuscript preparation. Jennifer Kane, MD: equally contributed to this work and aided in acquisition and analysis of the patient's data as well as manuscript preparation. Leslie Robinson-Bostom, MD: aided in the analysis and interpretation of the patient's data including histopathologic and Gram stain findings. She also aided in manuscript revision. John Kawaoka, MD: helped acquire and analyze patient information and aided in manuscript revision. $\mathrm{Na}$ thaniel Jellinek, MD: helped acquire data through nail biopsy and aided in the analysis and manuscript revision.

\section{References}

1 Negroni P. [Erythrasma of the nails]. Med Cutan Ibero Lat Am. 1976;4(5):349-57.

2 Duhard E, Calvet C, Mariotte N, Tichet J, Vaillant L. [Prevalence of longitudinal melanonychia in the white population]. Ann Dermatol Venereol. 1995;122(9):586-90.
3 Jellinek N. Nail matrix biopsy of longitudinal melanonychia: diagnostic algorithm including the matrix shave biopsy. J Am Acad Dermatol. 2007;56(5):803-10.

4 Haneke E, Baran R. Longitudinal melanonychia. Dermatol Surg. 2001;27(6):580-4.
5 Sommer LL, Reboli AC, Heymann WR. Bacterial diseases. In: Bolognia JL, Schaffer JV, Cerroni L, editors. Dermatology. 4th ed. Philadelphia: Elsevier; 2018. Vol. 1; p. 1274-7.

6 Towersey L, Azulay RD, Soares Filho PJ, Fischman-Gompertz O, Hay RJ. Kytococcus sedentarius nail infection. J Am Acad Dermatol. 2008;58(2):AB88. 